\title{
Peripheral blood mononuclear cell gene expression profile in obese boys who followed a moderate energy-restricted diet: differences between high and low responders at baseline and after the intervention
}

\author{
Tara Rendo-Urteaga ${ }^{1}$, Sonia García-Calzón ${ }^{1} \dagger$, Pedro González-Muniesa ${ }^{1,2,3}$, Fermín I. Milagro ${ }^{1,2,3}$, \\ María Chueca $^{4}$, Mirentxu Oyarzabal ${ }^{4}$, M. Cristina Azcona-Sanjulián ${ }^{5}$, J. Alfredo Martínez ${ }^{1,2,3}$ and \\ Amelia Marti ${ }^{1,2}$ t* $^{*}$ \\ ${ }^{1}$ Department of Nutrition, Food Science and Physiology, University of Navarra, C/Irunlarrea s/n, CP31008 Pamplona, \\ Navarra, Spain \\ ${ }^{2}$ CIBER Fisiopatología de la Obesidad y Nutrición (CIBERobn), Instituto de Salud Carlos III, Madrid, Spain \\ ${ }^{3}$ Centre for Nutrition Research, University of Navarra, Pamplona, Spain \\ ${ }^{4}$ Paediatric Endocrinology Unit, Complejo Hospitalario de Navarra, Pamplona, Spain \\ ${ }^{5}$ Paediatric Endocrinology Unit, Department of Paediatrics, Clinica Universidad de Navarra, Pamplona, Spain
}

(Submitted 9 November 2013 - Final revision received 4 August 2014-Accepted 16 October 2014 - First published online 4 December 2014)

\section{Abstract}

The present study analyses the gene expression profile of peripheral blood mononuclear cells (PBMC) from obese boys. The aims of the present study were to identify baseline differences between low responders (LR) and high responders (HR) after 10 weeks of a moderate energy-restricted dietary intervention, and to compare the gene expression profile between the baseline and the endpoint of the nutritional intervention. Spanish obese boys (age 10-14 years) were advised to follow a 10-week moderate energy-restricted diet. Participants were classified into two groups based on the association between the response to the nutritional intervention and the changes in BMI standard deviation score (BMI-SDS): HR group ( $n$ 6), who had a more decreased BMI-SDS; LR group ( $n$ 6), who either maintained or had an even increased BMI-SDS. The expression of 28869 genes was analysed in PBMC from both groups at baseline and after the nutritional intervention, using the Affymetrix Human Gene 1.1 ST 24-Array plate microarray. At baseline, the HR group showed a lower expression of inflammation and immune response-related pathways, which suggests that the LR group could have a more developed pro-inflammatory phenotype. Concomitantly, LEPR and SIRPB1 genes were highly expressed in the LR group, indicating a tendency towards an impaired immune response and leptin resistance. Moreover, the moderate energy-restricted diet was able to down-regulate the inflammatory 'mitogen-activated protein kinase signalling pathway' in the HR group, as well as some inflammatory genes (AREG and TNFAIP3). The present study confirms that changes in the gene expression profile of PBMC in obese boys may help to understand the weightloss response. However, further research is required to confirm these findings.

\section{Key words: Obesity: Children: Microarrays: Weight loss}

The worldwide prevalence of childhood overweight and obesity is increasing rapidly ${ }^{(1)}$, and is expected to reach $9 \%$ in $2020^{(1)}$. In Spain, the prevalence of overweight is about $45 \%$ in children aged $6-9$ years ${ }^{(2,3)}$. Young people with excessive body weight are more likely to develop obesity-related co-morbid conditions at early ages ${ }^{(1,4)}$, mainly type 2 diabetes, $\mathrm{CVD}^{(5)}$, hypertension and others ${ }^{(6,7)}$. In this sense, obesity is viewed as one of the major current public health problems with a high impact on children, leading to significant morbidity in adulthood ${ }^{(8)}$. Treating obesity in children and adolescents is critical to prevent obesity-related complications in adults ${ }^{(9)}$. Small reductions in body weight (between 0.25 and 0.50 BMI standard deviation score (BMI-SDS)) can trigger an improvement in metabolic outcomes in obese children ${ }^{(10-12)}$

Abbreviations: $A R E G$, amphiregulin; EGF, epidermal growth factor; HR, high responders; IGKC, Igא constant; JAM3, junctional adhesion molecule 3; KEGG, Kyoto Encyclopaedia for Genes and Genomes; LEPR, leptin receptor; LR, low responders; LTBP1, latent transforming growth factor $\beta$-binding protein 1 ; $M A P K$, mitogen-activated protein kinase; MMRN1, multimerin 1; PBMC, peripheral blood mononuclear cells; PKHD1L1, polycystic kidney and hepatic disease 1-like 1; RT-PCR, real-time PCR; SDS, standard deviation score; SELP, selectin P; SIRPB1, signal-regulatory protein $\beta 1$; TFPI, tissue factor pathway inhibitor; WL, weight loss.

*Corresponding author: Dr A. Marti, fax +34948 425619, email amarti@unav.es

†These authors contributed equally to this work. 
The use of blood cells for gene expression studies offers an advantage over the use of other human tissues because they are the most readily accessible tissue and are therefore of great interest for clinical studies and experimental research ${ }^{(13,14)}$. Moreover, the use of these cells for gene expression studies is a non-invasive technique, which offers an advantage for clinical studies. Specifically, the possibility of using gene expression measures in blood cells as the markers of metabolic status in obese children has been indicated ${ }^{(15)}$. Blood cells are being used to assess biological responses, as gene expression profiles may reflect the pathological and physiological states of the organism $^{(15)}$. Previous microarray studies in human subjects have shown changes in gene expression profiles after a lowenergy dietary intervention ${ }^{(16)}$, aimed to identify target genes associated with obesity that may help to develop personalised dietary treatments ${ }^{(17)}$. Peripheral blood mononuclear cells (PBMC) have been used in other studies investigating gene expression profiles in children ${ }^{(18,19)}$; however, no results for after weight-loss (WL) intervention programmes in obese children are available.

The analysis of individual gene responses using techniques such as microarrays is becoming a powerful tool that plays a role in understanding the mechanisms that control the physiological response of immune cells to a variety of perturbations, but the enormity of the data generated by such analyses can be perplexing, and at the same time, it is increasingly recognised that the functional and physiological significance of changes in gene expression may be better understood by examining the coordinated modulation of groups of genes acting in discrete pathways. In general, very little is yet known about the individual expression of genes in mononuclear cells in response to the dietary intervention, and even less about specific gene profiles.

In the present study, the effects of a moderate energyrestricted diet on the gene expression profile of PBMC were examined in obese boys. We hypothesised that a moderate energy-restricted diet could promote the changes in the gene expression of PBMC in the population under study. In particular, the aim of the present study was to characterise the changes in the expression of genes in PBMC after a nutritional intervention, specifically between the highresponder (HR) and low-responder (LR) groups at (1) baseline and (2) after the nutritional intervention.

\section{Subjects and methods}

\section{Subjects}

In the present study, seventy-one children aged 7-15 years who were classified as overweight or obese according to the criteria of Cole et al. ${ }^{(20)}$ were invited to participate in an informative session. Children were recruited from the Endocrinology Paediatric Units of the University of Navarra Clinic and Navarra's Hospital Complex in Pamplona, Navarra. All of them were Spanish or schooling foreigners for at least 1 year in Spain. Participants with a major psychiatric illness, significant neurological disease, eating disorders, familial hyperlipidaemia or any sort of either major cardiovascular or respiratory complication were excluded. The present study was conducted according to the guidelines laid down in the Declaration of Helsinki, and all procedures involving the subjects were approved by the Ethics Committee of the University of Navarra. Written informed consent was obtained from all the children and their parents.

Among the initial seventy-one volunteers, fifty-four successfully underwent baseline anthropometric measurements, of which forty-four (twenty-two boys and twenty-two girls) concluded the 10-week dietary intervention (dropout rate $18.5 \%$; see Fig. 1), as published elsewhere ${ }^{(21,22)}$. The BMISDS was calculated as a function of the degree of obesity of the subjects when compared with BMI local reference standards $^{(23)}$. The response of the participants to the intervention programme was based on the changes in BMI-SDS. After dichotomising the sample at the median (equal to $0.5 \mathrm{BMI}-$ SDS), participants were divided into two groups: HR group ( $n$ 6), who had a more decreased BMI-SDS; LR group, who either maintained or had an even increased BMI-SDS (Fig. 1).

Previous studies have shown that an improvement in body composition and cardiometabolic risk can be achieved with a BMI-SDS reduction of $\geq 0.25$ in obese adolescents, but greater benefits occur when losing at least 0.5 BMI-SDS ${ }^{(11)}$.

\section{Dietary intervention}

Dietary follow-up, weight control and nutritional education were performed weekly through individual sessions with a registered dietitian. Obese boys from the HR and LR groups with their father, mother or tutor - did similarly attend the appointments. Subjects were prescribed a fixed full-day meal diet, calculated according to the BMR. Energy expenditure was estimated taking into account basal metabolism using the Schofield equation, according to $\operatorname{sex}^{(24)}$ and body growth rate ${ }^{(25)}$. Moderate energy restriction was calculated according to the children's obesity degree ${ }^{(26)}$, not taking into account the body growth rate. In all cases, diets were not lower than $5439 \cdot 2 \mathrm{~kJ} / \mathrm{d}(1300 \mathrm{kcal} / \mathrm{d})$ and not higher than

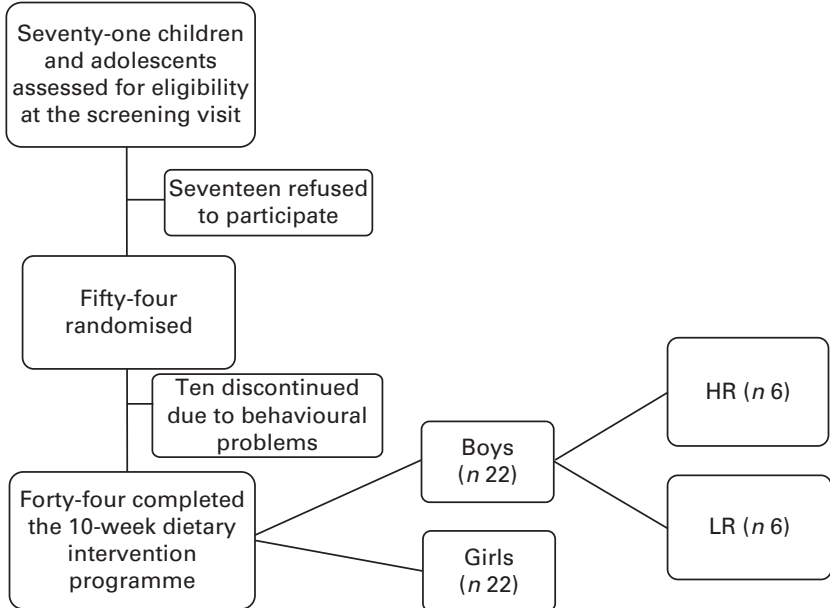

Fig. 1. Flow diagram describing the recruitment, study flow and follow-up of the participants. We chose six boys who had a decreased BMI-standard deviation score (SDS) as the high-responder (HR) group, and six boys who either maintained or had an increased BMI-SDS as the low-responder (LR) group. 
$9204.8 \mathrm{~kJ} / \mathrm{d}(2200 \mathrm{kcal} / \mathrm{d})$. Participants and their parents received personal training in nutritional and physical education throughout the whole intervention period. A semi-quantitative FFQ, previously validated in Spain ${ }^{(27,28)}$, containing 132 food items was filled in to evaluate dietary patterns in the obese children under study.

\section{Anthropometric, clinical and biochemical measurements}

Weight and height were measured with an electronic scale (Type SECA 861; Seca) and a telescopic height-measuring instrument (Type SECA 225; Seca), respectively. Waist and hip circumferences were measured with a flexible non-stretchable measuring tape (Type SECA 200; Seca). Body fat mass was estimated by bioelectrical impedance analysis (TBF-410; Tanita, Inc.). Blood pressure measurement was obtained from the left arm after a $15 \mathrm{~min}$ rest using a blood pressure monitor (OMRON M6; OMRON Healthcare Europe B.V.). Glucose, insulin and lipid profiles were determined by standard autoanalyser techniques. Insulin resistance was calculated from fasting glucose and insulin levels by using the homeostasis model assessment of insulin resistance ${ }^{(29)}$. Also, the quantitative insulin sensitivity check index method was used for the calculation of insulin sensitivity ${ }^{(30)}$. All parameters were measured at baseline and after the nutritional intervention.

\section{Extraction and isolation of RNA from peripheral blood mononuclear cells}

Gene expression analysis was carried out in PBMC from twelve obese boys (six HR and six LR) at baseline and after the nutritional intervention. PBMC were obtained from $10 \mathrm{ml}$ EDTA anti-coagulated peripheral blood by density centrifugation at $450 \mathrm{~g}$ for $30 \mathrm{~min}$ at $21^{\circ} \mathrm{C}$ using a Polymorphrep procedure (Axis-Shield PoC AS).

Total RNA was isolated from PBMC using the TRIzol method according to the manufacturer's instructions (Invitrogen). Extracted RNA was purified using the RNeasy Mini kit (Qiagen). RNA concentrations and purity were determined using a NanoDrop ND-1000 spectrophotometer (NanoDrop Technologies). RNA quality was assessed on an Agilent 2100 Bioanalyser, yielding a number of RNA integrity higher than 8.0 in all the samples.

\section{Synthesis of complementary DNA}

RNA (about $300 \mathrm{ng}$ ) was converted into complementary DNA following the manufacturer's instructions (Ambion ${ }^{\circledR}$ WT Expression kit; Affymetrix UK Ltd). Then, each aliquot of complementary DNA was fragmented using UDG (Uracil DNA Glycosylase) and APE1 (apurinic/apyrimidinic endonuclease 1), and labelled with terminal transferase using the Whole-Transcript Terminal Labelling kit of Affymetrix.

\section{Hybridisation and scanning}

Gene expression was analysed by microarray hybridisation in Progenika Biopharma, S.A. The Human Gene 1.1 ST 24-Array
Plate (Affymetrix) was used the microarray, which analyses 28869 genes with about twenty-six probes per gene.

The hybridisation, wash, developing and scanning of the samples was performed using a GeneTitan ${ }^{\circledR}$ platform (Affymetrix), according to the manufacturer's recommendations.

\section{Quantitative assessment of gene expression by real-time PCR}

To confirm the findings on gene expression microarray data, a real-time PCR (RT-PCR) protocol was performed with the TaqMan Universal PCR Master Mix and using an ABI PRISM 7900HT Fast Real-Time PCR System (Applied Biosystems). The expression of both target and housekeeping (cyclophilin A) genes for each sample was quantified based on their respective threshold cycle value $\left(\mathrm{dd} C_{\mathrm{t}}\right)$. The threshold cycle value of target genes was normalised to that of cyclophilin $\mathrm{A}$, and then expressed as the fold change (FC). Primer references obtained from Applied Biosystems are indicated as follows: signalregulatory protein $\beta 1$ (SIRPB1; NM_006065; Hs03645221_m1); leptin receptor (LEPR; NM_002303; Hs00174497_m1); epidermal growth factor (EGF; NM_001963; Hs00196731_ $\mathrm{m} 1$ ); tissue factor pathway inhibitor (TFPI; NM_006287; Hs01099999_m1); junctional adhesion molecule 3 (JAM3; NM_032801; Hs00230289_m1); latent transforming growth factor $\beta$-binding protein 1 (LTBP1; NM_206943; Hs0038 6448_m1); multimerin 1 (MMRN1; NM_007351; Hs0020 1182_m1); polycystic kidney and hepatic disease 1-like 1 (PKHD1L1; NM_177531; Hs00415429_m1); selectin P (SELP; NM_003005; Hs00927900_m1); IgK constant (IGKC; BC07 3772; Hs02384840_gH); amphiregulin (AREG; NM_001657; Hs00950669_m1); heparin-binding EGF-like growth factor (HBEGF; NM_001945; Hs00181813_m1); nuclear receptor subfamily 4, group A, member 2 (NR4A2; NM_006186; Hs004 28691_m1); TNF $\alpha$-induced protein 3 (TNFAIP3; NM_006290; Hs00234713_m1). A comparative study of the following genes was performed, with cyclophilin A being used as a stable internal marker: $R N A$; $28 \mathrm{~S}$ ribosomal 1 (RN28S1); actin $\beta$ (ACTB); glyceraldehyde 3-phosphate dehydrogenase (GAPDH); phosphoglycerate kinase 1 (PGK1). These genes were measured by RT-PCR in the samples obtained from the obese children under study before and after the WL programme. The expression levels of cyclophilin A mRNA (Hs04194521_s1) were not found to be affected by the experimental conditions and thus was used as the housekeeping gene. In this sense, cyclophilin A has previously been adopted as a housekeeping gene in PBMC experiments ${ }^{(31,32)}$.

\section{Statistical analyses}

Statistical analyses were performed using SPSS for Windows 15.0 software (SPSS, Inc.). For all the analyses, the significance level was set at $\alpha=0.05$, and all the tests were two-sided. The sample size calculation indicated that five subjects per group were required to be included in the study. This estimation was based on the following assumptions: an $\alpha$ error of $5 \%$, a power of $80 \%$, and a mean difference of 0.50 (SEM $0 \cdot 27$ ) units in BMI-SDS after the nutritional intervention. 
The Shapiro-Wilk test was used to determine variable distributions. Non-normally distributed variables (insulin levels) were log-transformed before an appropriate application of parametric statistical tests. An unpaired $t$ test was used to assess the differences in anthropometric and metabolic characteristics between the HR and LR groups at baseline. A paired $t$ test was used to compare the changes in both groups based on the nutritional intervention (before $v$. after intervention). A two-way ANOVA (response and time) was performed to compare the changes in the expression levels of genes from the microarray data between the HR and the LR groups at baseline and after 10 weeks of the nutritional intervention. In addition, a linear regression model was fitted to assess the changes in anthropometric variables according to basal gene expression levels. The respective value of the metabolic covariate was adjusted at baseline (after controlling for metabolic variables), and $\mathrm{B}$ regression coefficient values and 95\% CI were calculated.

\section{Microarray data analysis}

For the analysis, microarray raw files were imported into Partek GS (Partek, Inc.). Feature intensity values were normalised and reduced to expression summaries using the Robust Multichip Average method ${ }^{(33)}$. Probe sets were filtered based on raw intensity values by setting an arbitrary threshold of 100 fluorescence units to remove those with near-to-background values. In addition, probe sets that remained unchanged across the experiment were also removed from further analysis using the standard deviation of normalised intensity. A total of 10173 genes passed these filters. Differentially expressed genes were detected using repeated-measures ANOVA, setting the significance threshold at $P<0.05$ ( $n 2173$ genes). Multiple testing was controlled after the application of Bonferroni (data not shown) and false discovery rate corrections. The results were annotated using DAVID (Database for Annotation, Visualization and Integrated Discovery) software ${ }^{(34-36)}$, and pathway analysis was conducted using the Kyoto Encyclopaedia of Genes and Genomes (KEGG) metabolic pathway database $^{(37)}$. Raw gene expression data obtained from the microarray data analysis are available at the Gene Expression Omnibus database $^{(21)}$ (accession no. GSE41505).

\section{Results}

The characteristics of the six obese boys who had a more decreased BMI-SDS (HR group), and the six boys who either maintained or had an even increased BMI-SDS (LR group) are summarised in Table 1. No differences in the characteristics, including the Tanner stage (data not shown), were found between the two groups at baseline. Food intake at baseline was found to be similar in both groups (HR $v$. LR), and after the 10-week intervention programme, a trend was observed towards the lower intake of total energy and macronutrients (Table 1), which was statistically significant only in the HR group $(P=0 \cdot 021)$.

The 10-week intervention programme had a beneficial effect on anthropometric and biochemical measurements (Table 1). Obese boys in the LR group did not achieve statistically significant WL, but were able to maintain their body weight. A significant decrease in serum HDL-cholesterol concentration was observed in the LR group after the nutritional intervention. In the HR group, the mean WL was found to be 4.47 (sem 0.23 ) $\mathrm{kg}(5.7 \%$ loss of initial body weight, $P<0.001$ ). Specifically, these individuals showed a significant reduction in BMI-SDS $(-20.6 \%, P=0.001)$ and waist circumference $(-6.5 \%, P=0.001)$ after the nutritional intervention. During the moderate energy-restricted diet intervention, WL was associated with an improvement in insulin sensitivity, as assessed by the quantitative insulin sensitivity check index $(6.7 \%, P<0.05)$ and the homeostasis model assessment index $(-44.3 \%, P=0.001)$.

With regard to the microarray data, forty-eight genes were overexpressed and seven genes repressed, after a statistical comparison of the expression profiles of PBMC between the LR and HR groups at baseline (FC $\pm 1.5, P<0.05$; see online supplementary Table S1(A)). Similarly, nine genes were upregulated and thirty-three down-regulated in the HR group after the nutritional intervention (FC $\pm 1.5, P<0.05$; see online supplementary Table S1(B)).

In addition, genes implicated in metabolism and obesityrelated alterations were carefully examined according to the literature, since the aim of the study was to characterise the response to $\mathrm{WL}$ in obese boys.

In total, the expression levels of ten target genes (EGF, TFPI, LEPR, JAM3, LTBP1, MMRN1, PKHD1L1, SELP, SIRPB1 and $I G K C$ ) were found to be similar in both groups at baseline, as assessed by the RT-PCR and microarray analysis. Specifically, the microarray data were summarised after the comparison between (1) the LR and HR groups at baseline and (2) before and after the nutritional intervention in the HR group.

\section{Comparison of low responders v. high responders at baseline}

At baseline, the gene expression profile of PBMC in the LR group compared with the HR group showed the overexpression of forty-eight genes and the repression of seven genes ( $\mathrm{FC} \pm 1 \cdot 5$, $P<0 \cdot 05$; Fig. 2(a); see also online supplementary Table S1(A)). Notably, three of the seven underexpressed genes in the LR group did correspond to IGKC ( -1.75 -fold), encoding the constant domain of $\kappa$-type light chains for antibodies. Several overexpressed genes in the LR group compared with the HR group were as follows: SIRPB1 (NM_006065; 4.09-fold); LEPR (1.50-fold); EGF (1.52-fold); TFPI (1.82-fold); JAM3 (2.09-fold); LTBP1 (1.69-fold); MMRN1 (1.87-fold); PKHD1L1 (1.87-fold); SELP (1.82-fold).

Moreover, a comparison of gene expression levels from the microarray data was made between the HR and LR groups at baseline and after 10 weeks of the nutritional intervention using the two-way ANOVA (response, time; Fig. 3). There were statistically significant changes in the expression levels of the ten target genes between the LR and HR groups $\left(P_{\text {response }}\right)$. The transcript levels of $I G K C$ were significantly higher in the HR group, whereas those of the other nine genes were underexpressed in the HR group compared with the LR group (Fig. 3). However, the effect of the intervention 
Table 1. Characteristics of the obese boys at baseline and after 10 weeks of the nutritional intervention according to the diet response (Mean values with their standard errors)

\begin{tabular}{|c|c|c|c|c|c|c|c|c|c|c|c|c|}
\hline & \multicolumn{5}{|c|}{$\mathrm{HR}(n 6)$} & \multicolumn{5}{|c|}{$\operatorname{LR}(n 6)$} & \multirow[b]{3}{*}{$P \dagger$} & \multirow[b]{3}{*}{$P \ddagger$} \\
\hline & \multicolumn{2}{|c|}{ Baseline } & \multicolumn{2}{|c|}{ After intervention } & \multirow[b]{2}{*}{$P^{\star}$} & \multicolumn{2}{|c|}{ Baseline } & \multicolumn{2}{|c|}{ After intervention } & \multirow[b]{2}{*}{$P^{*}$} & & \\
\hline & Mean & SEM & Mean & SEM & & Mean & SEM & Mean & SEM & & & \\
\hline Age (years) & $13 \cdot 17$ & 0.98 & & & & $12 \cdot 83$ & 1.60 & & & & 0.673 & \\
\hline Weight $(\mathrm{kg})$ & 79.02 & 3.69 & 74.55 & 3.71 & $<0.001$ & 89.55 & $6 \cdot 35$ & $90 \cdot 30$ & 6.68 & 0.247 & 0.182 & 0.066 \\
\hline Height $(m)$ & 1.62 & 0.03 & 1.63 & 0.03 & 0.019 & 1.62 & 0.04 & 1.64 & 0.04 & 0.038 & 0.936 & 0.885 \\
\hline BMI-SDS & $3 \cdot 10$ & 0.54 & 2.46 & 0.48 & 0.001 & 4.08 & 0.43 & 4.01 & 0.46 & 0.421 & 0.185 & 0.042 \\
\hline Body fat (\%) & 29.57 & 3.66 & 27.08 & 2.31 & 0.245 & 33.82 & 1.70 & 33.45 & 1.59 & 0.608 & 0.327 & 0.047 \\
\hline Waist circumference $(\mathrm{cm})$ & 96.00 & 1.52 & 89.75 & 1.96 & 0.001 & 99.67 & 2.82 & 98.80 & 3.43 & 0.691 & 0.280 & 0.045 \\
\hline Hip circumference $(\mathrm{cm})$ & $102 \cdot 70$ & 1.71 & 99.13 & $1 \cdot 70$ & 0.001 & $108 \cdot 72$ & $2 \cdot 73$ & $107 \cdot 08$ & 3.04 & 0.089 & 0.091 & 0.046 \\
\hline Waist:hip ratio & 0.94 & 0.01 & 0.91 & 0.01 & 0.028 & 0.92 & 0.02 & 0.92 & 0.02 & 0.749 & 0.463 & 0.469 \\
\hline Waist:height ratio & 0.59 & 0.01 & 0.55 & 0.01 & 0.001 & 0.61 & 0.01 & 0.60 & 0.02 & 0.501 & 0.278 & 0.029 \\
\hline $\mathrm{DBP}(\mathrm{mmHg})$ & 69.67 & 3.57 & 70.67 & $5 \cdot 65$ & 0.906 & 76.50 & 7.73 & 73.50 & 3.77 & 0.735 & 0.441 & 0.685 \\
\hline $\mathrm{SBP}(\mathrm{mmHg})$ & $135 \cdot 50$ & 10.09 & $127 \cdot 17$ & 6.48 & 0.476 & 134.33 & 4.03 & $136 \cdot 83$ & 6.35 & 0.739 & 0.917 & 0.312 \\
\hline Heart rate (beats/min) & $81 \cdot 20$ & 2.69 & 73.80 & 4.49 & 0.051 & $76 \cdot 25$ & 1.55 & 71.50 & 1.44 & 0.050 & 0.182 & 0.520 \\
\hline Serum glucose $(\mathrm{mmol} / \mathrm{l})$ & $5 \cdot 40$ & 0.14 & 4.99 & 0.20 & 0.076 & 5.09 & 0.10 & 4.83 & 0.12 & 0.079 & 0.107 & 0.524 \\
\hline Serum insulin $(\mu \mathrm{U} / \mathrm{ml}) \S$ & 1.34 & 0.02 & $1 \cdot 16$ & 0.07 & 0.043 & 1.35 & 0.10 & 1.37 & 0.11 & 0.713 & 0.910 & 0.136 \\
\hline HOMA-IR & $5 \cdot 24$ & 0.34 & 2.92 & 0.50 & 0.001 & 5.90 & 1.39 & 5.91 & 1.51 & 0.984 & 0.660 & 0.197 \\
\hline QUICKI & 0.30 & 0.00 & 0.32 & 0.01 & 0.034 & 0.303 & 0.01 & 0.303 & 0.01 & 1.000 & 0.763 & 0.162 \\
\hline Serum total cholesterol $(\mathrm{mmol} / \mathrm{l})$ & 8.43 & 0.70 & 7.85 & 0.70 & 0.308 & 9.00 & 0.44 & 8.98 & 0.48 & 0.896 & 0.512 & 0.212 \\
\hline Serum TAG (mmol/l) & $1 \cdot 24$ & 0.25 & 0.85 & 0.20 & 0.125 & $1 \cdot 16$ & 0.38 & 1.58 & 0.56 & 0.246 & 0.863 & 0.263 \\
\hline Serum HDL-cholesterol (mmol/l) & 2.09 & 0.16 & 2.02 & 0.11 & 0.634 & $2 \cdot 62$ & 0.27 & $2 \cdot 31$ & 0.22 & 0.018 & 0.113 & 0.299 \\
\hline Serum LDL-cholesterol (mmol/l) & 5.08 & 0.75 & 4.87 & 0.73 & 0.580 & $5 \cdot 18$ & 0.40 & 4.93 & 0.37 & 0.426 & 0.873 & 0.932 \\
\hline Cholesterol:HDL-cholesterol ratio & $4 \cdot 20$ & 0.57 & $4 \cdot 10$ & 0.60 & 0.697 & 3.67 & 0.48 & 4.08 & 0.48 & 0.107 & 0.472 & 0.983 \\
\hline Energy intake $(\mathrm{kJ} / \mathrm{d})$ & $11022 \cdot 74$ & 902.99 & 8176.66 & 1551.43 & 0.021 & $10397 \cdot 24$ & 1873.34 & 8067.84 & $1339 \cdot 17$ & 0.462 & 0.771 & 0.959 \\
\hline Carbohydrate intake $(\mathrm{g} / \mathrm{d})$ & 293.05 & 37.97 & 221.99 & 46.04 & 0.047 & 282.05 & 55.66 & $224 \cdot 21$ & 39.73 & 0.618 & 0.763 & 0.792 \\
\hline Protein intake $(\mathrm{g} / \mathrm{d})$ & $118 \cdot 17$ & 7.69 & 98.54 & 16.53 & 0.192 & 108.07 & 16.95 & 83.97 & $8 \cdot 16$ & 0.346 & 0.602 & 0.452 \\
\hline Lipid intake (g/d) & 109.96 & 10.55 & 74.68 & $15 \cdot 38$ & 0.061 & $107 \cdot 17$ & $25 \cdot 27$ & $77 \cdot 28$ & $16 \cdot 10$ & 0.453 & 0.921 & 0.910 \\
\hline
\end{tabular}

HR, high responders; LR, low responders; SDS, standard deviation score; DBP, diastolic blood pressure; SBP, systolic blood pressure; HOMA-IR, homeostasis model assessment of insulin resistance; QUICKI, quantitative insulin sensitivity check index.

* $P$ value for the comparison between baseline and after the nutritional intervention in subjects distributed according to the response.

$\dagger P$ value for the comparison between the HR and LR groups at baseline.

$\ddagger P$ value for the comparison between the HR and LR groups after the nutritional intervention.

$\S$ Non-normally distributed variables were log-transformed before analysis. 
(a)

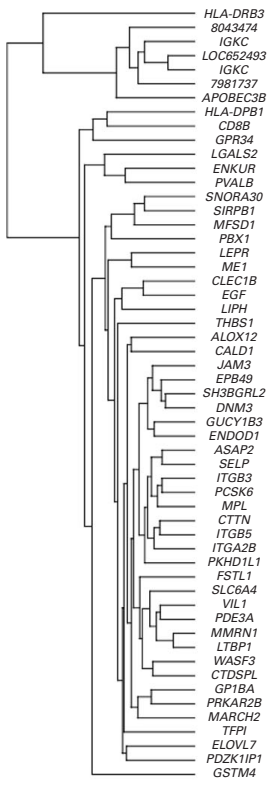

Hierarchical clustering

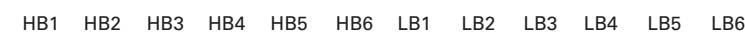

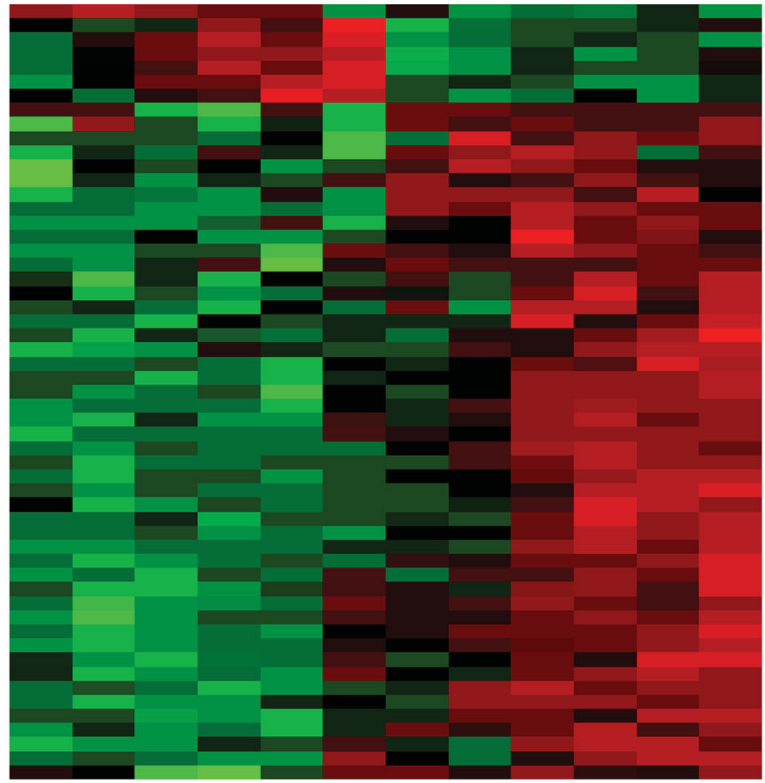

$-2.45$

$0 \cdot 00$

2.45

Hierarchical clustering

(b)

HB6 HB4 HB5 HB1 HB2 HB3 HA5 HA3 HA2 HA1 HA4 HA6
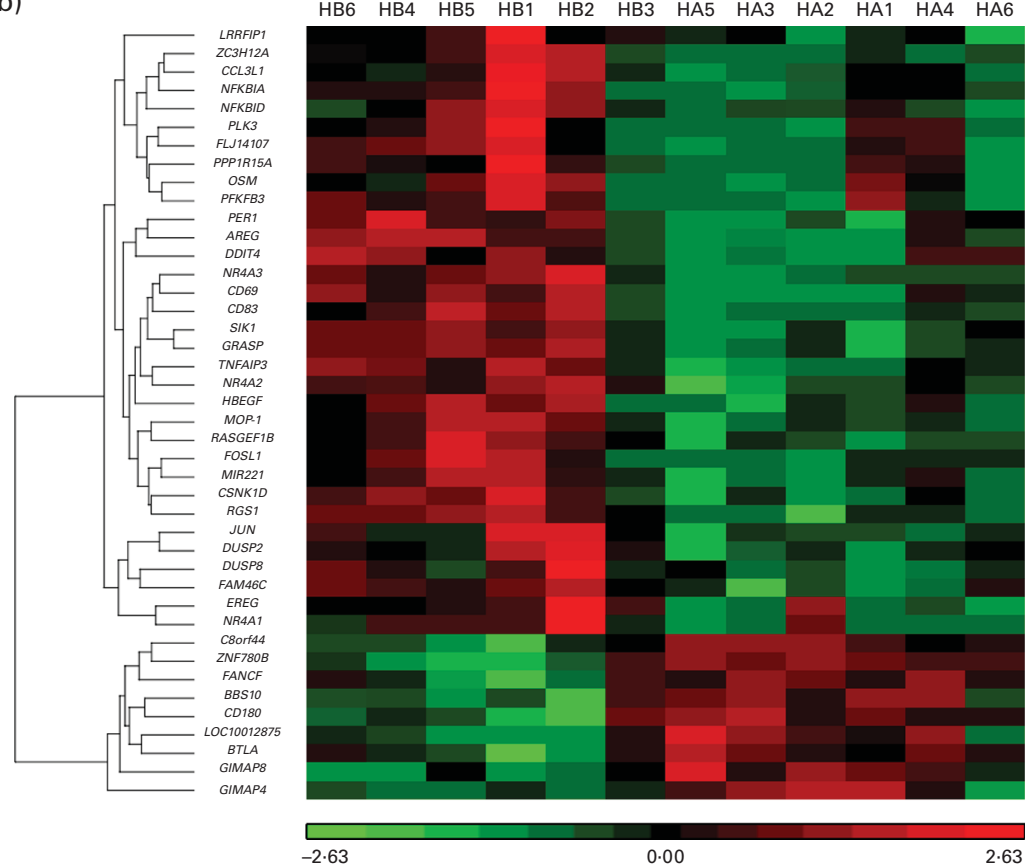

Fig. 2. Heat map analysis of microarray data showing (a) hierarchical clustering of fifty-five differentially expressed genes between the low-responder (LR) group and the high-responder (HR) group at baseline (LB and HB, respectively), and (b) hierarchical clustering of forty-two differentially expressed genes in the HR group before $(\mathrm{HB})$ and after (HA) the 10-week nutritional intervention programme. The red or green colours indicate differentially up- or down-regulated genes, respectively (1.5-fold change, $P<0.05)$, in the samples from twelve obese boys from the two groups (HR and LR), (b) before and (a) after the intervention. The comparison of (a) HB v. LB and (b) HA v. HB is shown. For a list of gene names and abbreviations, see online supplementary Table S1. (A colour version of this figure can be found online at http://www.journals.cambridge.org/bjn).

(before $v$. after, $P_{\text {time }}$ ) did not reach statistical significance for any of the genes studied.

To confirm the microarray results, the expression levels of the ten target genes (EGF, TFPI, LEPR, JAM3, LTBP1, MMRN1, PKHD1L1, SELP, SIRPB1 and IGKC) were measured using
RT-PCR. The genes chosen were among the top ten genes with a higher FC of up-regulation. The only exceptions were $L E P R$ and EGF, which were chosen because of their key regulatory role in energy homeostasis. Additionally, within the top ten up-regulated genes, three (glutathione $S$-transferase 


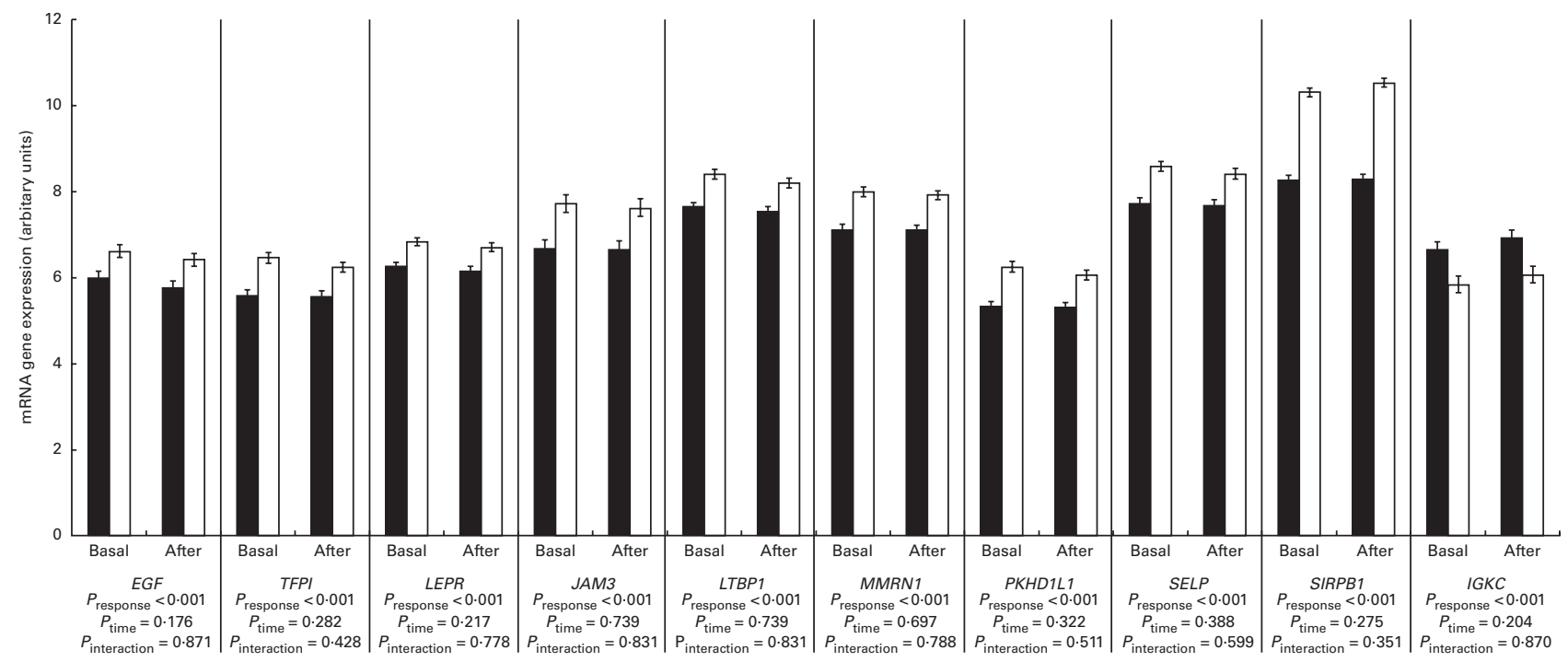

Fig. 3. Comparison of gene expression levels from the microarray data between the high-responder (HR, $\square$ ) group and low-responder (LR, $\square$ ) group at baseline and after 10 weeks of the nutritional intervention. Values are means, with their standard errors represented by vertical bars. Two-way ANOVA: $P_{\text {response, }}$

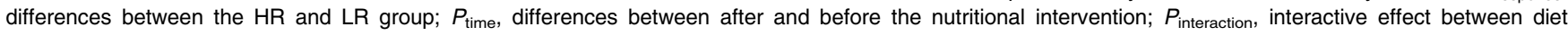
response and time. For a list of gene names and abbreviations, see online supplementary Table $\mathrm{S} 1$.

mu 4 (GSTM4), lectin, galactoside-binding, soluble, 2 (LGALS2) and major histocompatibility complex, class II, DP beta 1 (HLA-DPB1)) were not selected for quantitative PCR validation because of their higher $P$ value and false discovery rate, which offered less guarantee for the validation by the other technique. Among the down-regulated genes, IGKC was selected because of its highest change in the expression levels (FC -1.7). The changes in the expression levels of down-regulated genes were more modest (see online supplementary Table S1(A)) than those of the up-regulated genes, supporting the selection of only one gene in this category.

For all the genes, the direction and magnitude of the FC obtained from RT-PCR were comparable to those obtained from the microarray technique (Fig. 4(a)). Moreover, RT-PCR showed significant differences in five of the genes between the LR and HR groups $(P<0.05)$, and two of them almost reached statistical significance $(P<0 \cdot 1)$. Overall, most of the genes tested were validated by RT-PCR, suggesting that the microarray data represent true expression changes.

DAVID software ${ }^{(34)}$ was used to identify Gene Ontology biological processes and KEGG pathways. At baseline, nineteen biological processes showed significant differences between the LR and HR groups $(P<0.05$; see online supplementary Table S2), with platelet activation and blood coagulation being the most divergent processes. Moreover, nine KEGG pathways were significantly overexpressed in the LR group when compared with the HR group at baseline (Table 2), including extracellular matrix-receptor interaction, focal adhesion, cell adhesion molecules and IgG Fc receptor II (Fc $\gamma R$ )-mediated phagocytosis.

A linear regression model was fitted to predict the changes in anthropometric variables after the 10-week nutritional intervention programme, according to the baseline expression levels of the ten target genes (Table 3). Importantly, baseline transcript levels of EGF, TFPI, LEPR, JAM3, LTBP1, MMRN1,
PKHD1L1, SELP, SIRPB1 and LEPR were associated with the changes in body weight, BMI-SDS and body fat mass after the nutritional intervention, indicating that the lower the expression levels, the higher the decrease in adiposity indices.

\section{Comparison of before v. after the nutritional intervention in the high-responder group}

The nutritional intervention did alter the gene expression profile of PBMC, with a total of 2173 genes showing differential transcript levels $(P<0.05)$ in obese boys (Fig. 5). Specifically, approximately $28 \%$ of them (390 genes) were changed in both groups (HR and LR). In addition, 35.2\% (880 genes: 555 up-regulated and 325 down-regulated) were significantly modified in the HR group; meanwhile, 36.8\% (903 genes: 527 up-regulated and 376 down-regulated) were altered in the LR group

Specifically, nine genes were up-regulated and thirty-three down-regulated in the HR group (FC $\pm 1.5, P<0.05$ ) after the nutritional intervention (Fig. 2(b); see online supplementary Table S1(B)). AREG (-3.21-fold), HBEGF (-2.54-fold), NR4A2 (-2.49-fold) and TNFAIP3 (-2.13-fold) were among the down-regulated genes.

RT-PCR was performed to quantitatively measure the differences in the transcript levels of a select group of genes that were identified by the microarray technique. Similar to the microarray data, the decreased gene expression levels of AREG, HBEGF, NR4A2 and TNFAIP3 were also found by the RT-PCR technique, but without any statistical significance (Fig. 4(b)).

The Gene Ontology analysis revealed that twelve biological processes were significantly altered by the nutritional intervention in the HR group $(P<0.05$; see online supplementary Table S3). Among these biological processes, four were equally affected in the HR and LR groups: cellular response 

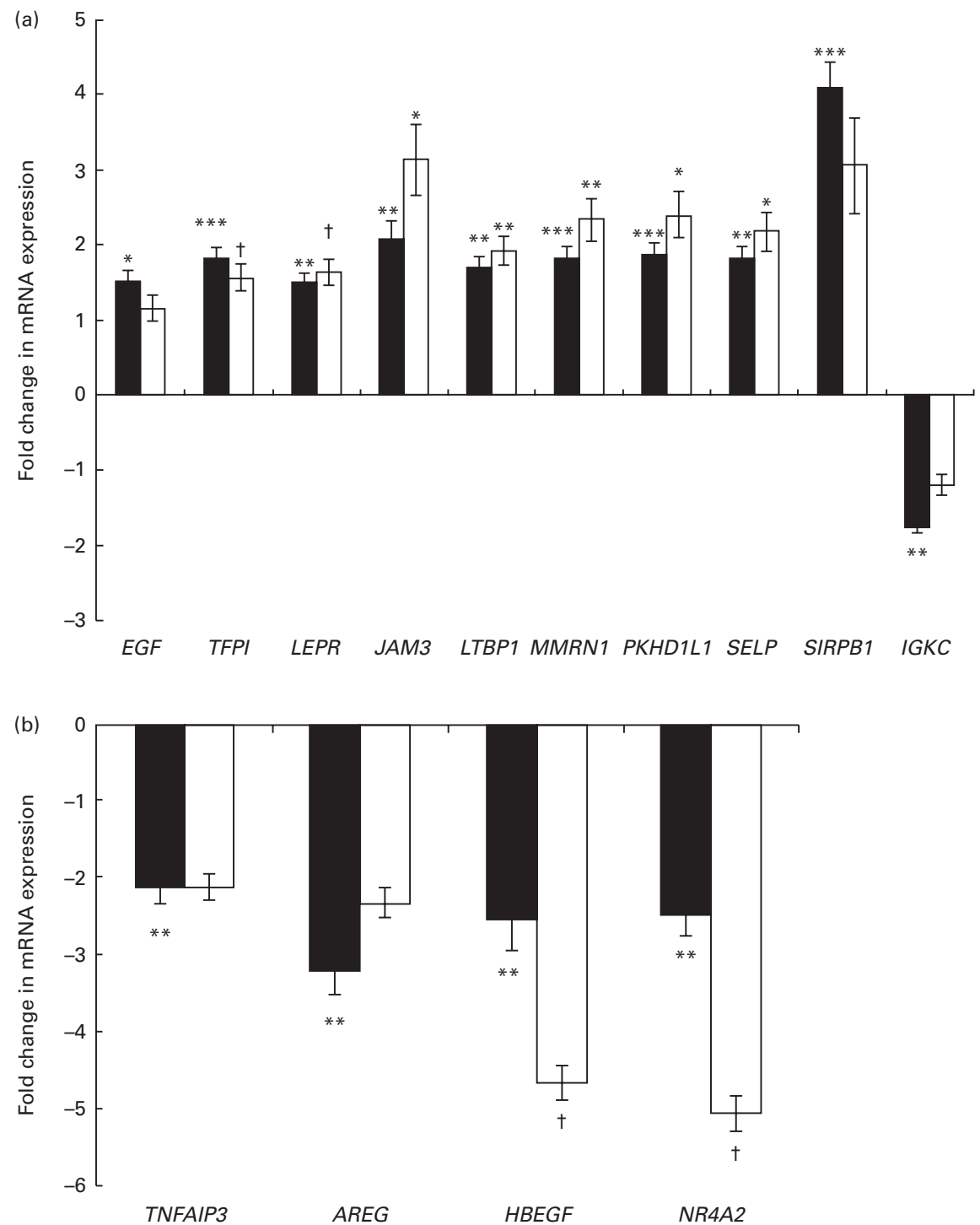

Fig. 4. Gene expression levels from the microarray $(\square)$ and real-time PCR $(\square)$ data. (a) Comparison between the low-responder (LR) and high-responder (HR) groups at baseline, and (b) comparison between baseline and after the moderate energy-restricted diet intervention in the HR group. Mean change compared with baseline was significant: ${ }^{*} P<0.05$, ${ }^{* *} P<0.01,{ }^{* *} P<0.001$. Mean change compared with baseline was marginally significant (trend): $\dagger P<0.10$. For a list of gene names and abbreviations, see online supplementary Table $\mathrm{S} 1$.

to stress; lymphocyte homeostasis; macromolecule catabolic process; negative regulation of NF-кB import into the nucleus. Interestingly, in the LR group, biological processes associated with immune, inflammatory and defence responses were significantly up-regulated after the nutritional intervention. According to the KEGG analysis, four pathways including several signalling pathways - erythroblastoma virus B receptor family (ErbB), epithelial cell, mitogen-activated protein kinases (MAPK) and circadian rhythm - were down-regulated after the nutritional intervention in the HR group (Table 2).

\section{Discussion}

In the present study, we examined the differences in gene expression levels and pathways in PBMC from obese boys, comparing the HR and LR groups at baseline and after a moderate energy-restricted diet intervention. To the best of our knowledge, the present study is the first to report on the microarray analyses of PBMC in obese boys. Although blood is usually not considered to be a target tissue for obesity, PBMC are relatively easily accessible in humans ${ }^{(37)}$ and are used to assess biological responses as their gene expression profile may reflect the pathological and physiological states of the organism ${ }^{(13)}$. It is important to point out that differences in gene expression levels due to nutritional interventions are usually small. However, changes in blood transcript levels in the HR and LR groups were in agreement with the RT-PCR results and informative enough to consider their analyses ${ }^{(16)}$. Interestingly, at baseline, the HR group showed a lower expression of inflammation and immune response-related pathways, which suggests that the LR group could have a more developed pro-inflammatory phenotype. This group 
Table 2. Overexpression of Kyoto Encyclopaedia for Genes and Genomes (KEGG) pathways in the lowresponder (LR) group compared with the high-responder (HR) group at baseline, and down-regulation in the HR group after the nutritional intervention

\begin{tabular}{|c|c|c|c|c|}
\hline Pathways (KEGG) & Genes $(n)$ & $P$ & Benjamini & ES \\
\hline \multicolumn{5}{|l|}{ Baseline } \\
\hline ECM-receptor interaction & 5 & $6.7 \times 10^{-4}$ & $3.8 \times 10^{-2}$ & 11.6 \\
\hline Haematopoietic cell lineage & 4 & $8.2 \times 10^{-3}$ & $2.1 \times 10^{-1}$ & $9 \cdot 1$ \\
\hline Focal adhesion & 5 & $1.6 \times 10^{-2}$ & $2.6 \times 10^{-1}$ & 4.9 \\
\hline Cell adhesion molecules & 4 & $2.6 \times 10^{-2}$ & $3.1 \times 10^{-1}$ & 5.9 \\
\hline Arrhythmogenic right ventricular cardiomyopathy & 3 & $5.3 \times 10^{-2}$ & $4.6 \times 10^{-1}$ & $7 \cdot 7$ \\
\hline Hypertrophic cardiomyopathy & 3 & $6.5 \times 10^{-2}$ & $4.7 \times 10^{-1}$ & 6.9 \\
\hline Dilated cardiomyopathy & 3 & $7.4 \times 10^{-2}$ & $4.7 \times 10^{-1}$ & 6.4 \\
\hline Fc $\gamma R$-mediated phagocytosis & 3 & $7.8 \times 10^{-2}$ & $4.4 \times 10^{-1}$ & $6 \cdot 2$ \\
\hline Regulation of actin cytoskeleton & 4 & $8.7 \times 10^{-2}$ & $4.4 \times 10^{-1}$ & $3 \cdot 6$ \\
\hline \multicolumn{5}{|l|}{ Before $v$. after the nutritional intervention } \\
\hline ErbB signalling pathway & 4 & $1.9 \times 10^{-3}$ & $5.4 \times 10^{-2}$ & $14 \cdot 6$ \\
\hline Epithelial cell signalling in Helicobacter pylori infection & 3 & $1.7 \times 10^{-2}$ & $2.1 \times 10^{-1}$ & $14 \cdot 0$ \\
\hline Circadian rhythm & 2 & $3.8 \times 10^{-2}$ & $3.1 \times 10^{-1}$ & $48 \cdot 9$ \\
\hline MAPK signalling pathway & 4 & $4.1 \times 10^{-2}$ & $2.6 \times 10^{-1}$ & 4.8 \\
\hline
\end{tabular}

ES, enrichment score; ECM, extracellular matrix; Fc $\gamma$ R, IgG Fc receptor II; ErbB, erythroblastoma virus B receptor family; MAPK mitogen-activated protein kinases.

presented higher expression levels of LEPR and SIRPB1, which indicates a tendency towards an impaired immune response and leptin resistance.

Moreover, the baseline expression levels of six genes (TFPI, LEPR, LTBP1, MMRN1, PKHD1L1 and SIRBP1) were consistently associated with the changes in adiposity indices (BMI-SDS).

There has not been much research on gene expression levels after a WL programme in obese children. However, in obese adults, there have been studies investigating the effect of energy restriction on the changes in gene expression. Most of these studies were performed in adipose tissue biopsies (eleven studies), but some in skeletal muscle (two studies) and PBMC (five studies) ${ }^{(3)}$. Unfortunately, they did not mention any changes in the fourteen genes that we characterised in the present study. Differences in study design, type of intervention, characteristics of subjects (age, sex, lifestyle factors, etc.), analytical procedure and so on may explain the discrepancies.
There are some limitations in the present study. The first limitation is the small sample size due to the significant costs involved in the microarray experiment. Second, participants were only males; in addition, there was no sample available to investigate the changes in the gene expression levels of PBMC after a WL programme in obese children. However, the present study reveals novel features regarding transcriptional changes in obese boys after a moderate energy-restricted diet intervention.

\section{Comparison of low responders v. high responders at baseline}

The KEGG analysis identified several pathways related to the overexpression of genes in the LR group at baseline, such as extracellular matrix-receptor interaction, focal adhesion and cell adhesion molecules. The first two pathways are associated with cell motility, proliferation, differentiation and survival;

Table 3. Association between baseline gene expression levels of the ten selected target genes and changes in adiposity indices after the 10 -week nutritional intervention*

(B regression coefficient values and $95 \%$ confidence intervals, $n$ 12)

\begin{tabular}{|c|c|c|c|c|c|c|c|c|c|}
\hline \multirow[b]{2}{*}{ Baseline gene expression } & \multicolumn{2}{|c|}{ Body-weight change (kg) } & \multirow[b]{2}{*}{$P$} & \multicolumn{2}{|c|}{ BMI-SDS change } & \multirow[b]{2}{*}{$P$} & \multicolumn{2}{|c|}{$\begin{array}{l}\text { Percentage of body fat } \\
\text { change }\end{array}$} & \multirow[b]{2}{*}{$P$} \\
\hline & $\mathrm{B}$ & $95 \% \mathrm{Cl}$ & & $\mathrm{B}$ & $95 \% \mathrm{Cl}$ & & B & $95 \% \mathrm{Cl}$ & \\
\hline$E G F$ & 3.60 & $0.70,6.50$ & 0.020 & 0.21 & $-0.38,0.80$ & 0.434 & $5 \cdot 88$ & $1.79,9.98$ & 0.010 \\
\hline TFPI & $4 \cdot 13$ & $2 \cdot 16,6 \cdot 10$ & 0.001 & 0.50 & $0.11,0.88$ & 0.017 & 3.52 & $0.15,6.89$ & 0.043 \\
\hline LEPR & 4.67 & $1.55,7.79$ & 0.008 & 0.59 & $0.02,1.16$ & 0.045 & 4.46 & $-0.09,9.01$ & 0.054 \\
\hline JAM3 & 3.06 & $1 \cdot 81,4 \cdot 31$ & $<0.001$ & 0.28 & $-0.04,0.60$ & 0.080 & $3 \cdot 16$ & $0.68,5.63$ & 0.019 \\
\hline$\angle T B P 1$ & 4.69 & $2 \cdot 58,6 \cdot 80$ & 0.001 & 0.57 & $0.12,1.01$ & 0.019 & $5 \cdot 98$ & $3 \cdot 76,8 \cdot 20$ & $<0.001$ \\
\hline MMRN1 & $4 \cdot 42$ & $2 \cdot 87,5.97$ & $<0.001$ & 0.53 & $0.13,0.92$ & 0.016 & $5 \cdot 22$ & $2 \cdot 64,7 \cdot 80$ & 0.002 \\
\hline PKHD1L1 & $4 \cdot 41$ & $2 \cdot 58,6 \cdot 25$ & 0.001 & 0.53 & $0.11,0.95$ & 0.019 & $5 \cdot 68$ & $3.54,7 \cdot 81$ & $<0.001$ \\
\hline SELP & 3.50 & $0.98,6.03$ & 0.013 & 0.36 & $-0.12,0.83$ & 0.122 & $5 \cdot 18$ & $2 \cdot 86,7.49$ & 0.001 \\
\hline SIRPB1 & $2 \cdot 24$ & $1 \cdot 36,3 \cdot 11$ & $<0.001$ & 0.28 & $0.10,0.46$ & 0.006 & 1.61 & $-0.05,3.28$ & 0.056 \\
\hline$I G K C$ & -3.02 & $-5.44,-0.60$ & 0.020 & -0.29 & $-0.75,0.17$ & $0 \cdot 185$ & -0.61 & $-4 \cdot 71,3 \cdot 49$ & 0.745 \\
\hline
\end{tabular}

SDS, standard deviation score; EGF, epidermal growth factor; TFPI, tissue factor pathway inhibitor; $L E P R$, leptin receptor; JAM3, junctional adhesion molecule 3; $L T B P 1$, latent transforming growth factor $\beta$-binding protein 1; MMRN1, multimerin 1; PKHD1L1, polycystic kidney and hepatic disease 1-like 1; SELP, selectin P; SIRPB1, signal-regulatory protein $\beta 1$; IGKC, Igк constant.

*Adjusted for the corresponding anthropometric variable at baseline. 


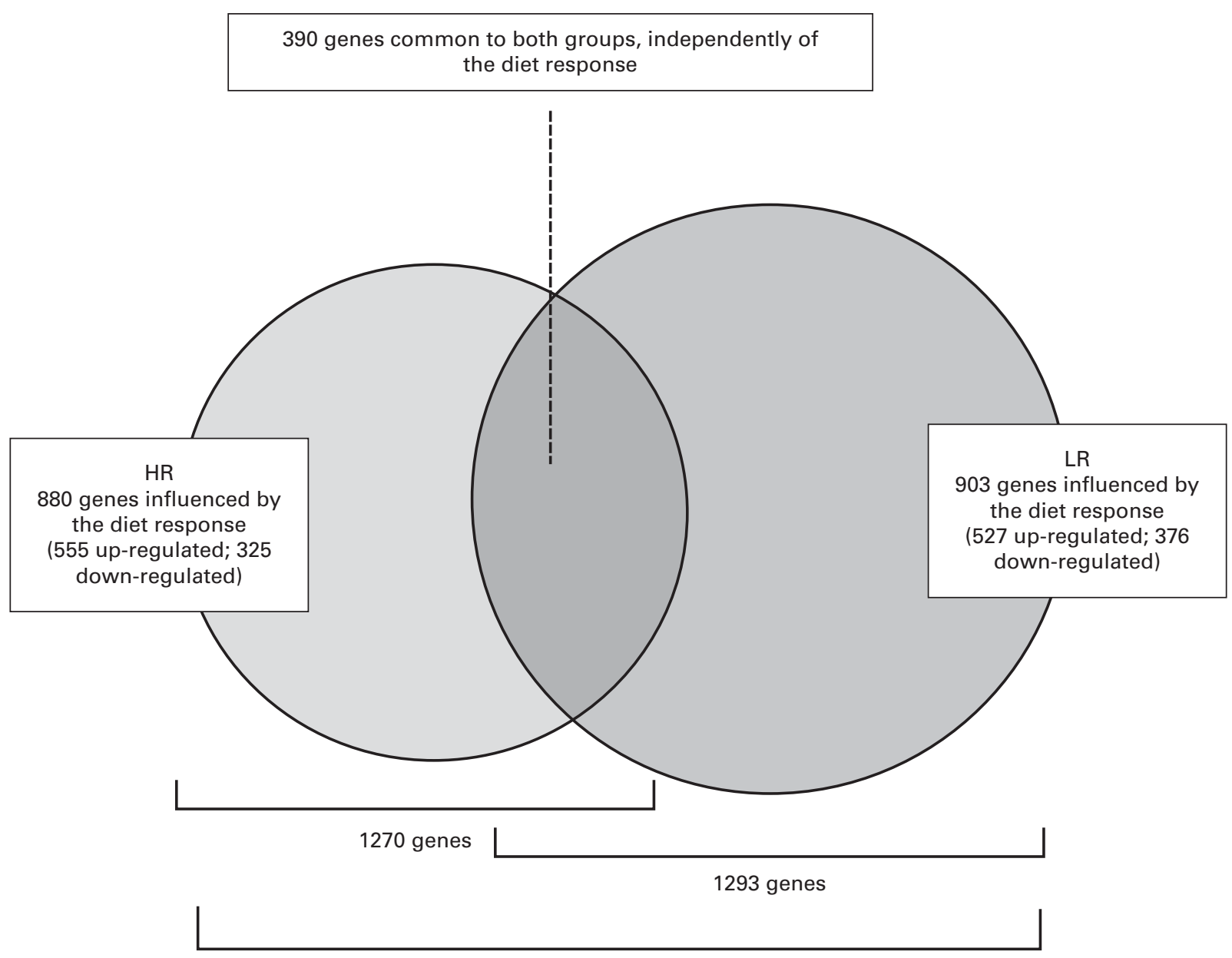

Total genes: 2173

Fig. 5. Comparison of the gene expression profile of peripheral blood mononuclear cells (PBMC) in obese boys according to the response (high-responder (HR) and low-responder (LR) groups) to a weight-loss (WL) programme, showing the relative magnitude of the effects (circles) and the size of the overlap (shaded area). A total of 390 PBMC genes were significantly altered by the WL programme in both groups.

meanwhile, cell adhesion molecules are linked to haemostasis, immune response and inflammation.

Another pathway related to the overexpression of genes in the LR group at baseline is 'Fc $\gamma \mathrm{R}$-mediated phagocytosis', which plays an essential role in host defence mechanisms through the uptake and destruction of infectious pathogens. Specialised cell types, including PBMC subsets such as macrophages, neutrophils and monocytes, take part in this process. In this sense, obesity has been associated with elevated leucocyte and lymphocyte subset counts and higher monocyte and granulocyte phagocytic activities ${ }^{(38)}$

Higher expression levels of SIRPB1 and $L E P R$ were found in the LR group at baseline. SIRPB1 belongs to the Ig superfamily and participates in macrophage activation. It is also overexpressed in the adipose tissue macrophages of diet-induced obese mice $^{(39)}$, and could be related to low-grade inflammation associated with obesity. This obesity-related inflammation status could impair the response to WL in the LR group. Similar results have been reported in blood cells from overweight children ${ }^{(15)}$. Moreover, TFPI and EGF genes were also overexpressed in the LR group. It has been reported that the TFPI gene is increased in patients with type 2 diabetes mellitus ${ }^{(40)}$, whereas the gene of the EGF receptor is up-regulated in obesity ${ }^{(41)}$.

\section{Comparison of before v. after intervention in the high-responder group}

The KEGG analysis identified four down-regulated pathways, including 'ErbB signalling pathway' and 'MAPK signalling pathway', in the HR group after the nutritional intervention. Both pathways are involved in processes that are associated with cell proliferation, differentiation, motility, migration and inflammation. The 'MAPK signalling pathway' plays an important role in inflammation ${ }^{(42)}$, and its inhibition could have anti-inflammatory properties. NR4A receptors may act as important transcriptional mediators of inflammatory signals, which are rapidly stimulated by the MAPK pathway ${ }^{(22)}$. These receptors have been associated with obesity in human adipose tissue, which are up-regulated in extreme obesity and normalised after fat loss ${ }^{(41)}$. They are mostly expressed in stromal vascular cells, a subset of cells that is rich in macrophages in obese adipose tissue ${ }^{(41)}$. 
In contrast, $A R E G$, a member of the $E G F$ gene family, is strongly down-regulated in PBMC after WL. $A R E G$ is pro-inflammatory ${ }^{(43)}$, and it may be hypothesised that its expression could be inhibited as a result of the reduction in obesity-linked inflammation. The other gene down-regulated after WL is $H B E G F$, which has been reported to be hypersecreted in obesity $^{(42)}$. It plays a role in insulin sensitivity, facilitating the use of peripheral glucose $\mathrm{e}^{(44)}$, and its expression is stimulated by leptin ${ }^{(45)}$. Thus, it could be hypothesised that, after the WL intervention, when leptin serum levels decrease, there may also be a decrease in the gene expression levels of $H B E G F$.

TNFAIP3, a key regulator of inflammation and immunity involved in the development of various autoimmune diseases ${ }^{(46)}$, is also down-regulated after WL. TNFAIP3 is a $\mathrm{Zn}$ finger protein whose expression is rapidly induced by $\mathrm{TNF}^{(47)}$. The nutritional intervention decreased the gene expression of TNFAIP3 in the HR group, which could be involved in the amelioration of obesityinduced inflammation and subsequent lower secretion of TNF $\alpha$.

In conclusion, the present study confirms that changes in the gene expression levels of PBMC in obese boys may help to understand the response to WL. Moreover, the present study suggests that the gene expression levels of TFPI, LEPR, LTBP1, MMRN1, PKHD1L1 and SIRBP1 at baseline could predict the changes in BMI-SDS after the nutritional intervention. However, more studies are needed to confirm these findings.

\section{Supplementary material}

To view supplementary material for this article, please visit http://dx.doi.org/10.1017/S0007114514003584

\section{Acknowledgements}

The authors thank all the children and their parents who participated in the study.

The present study was funded by grants from the Navarra Government, Departamento de Salud (grant no. PI 54/2009), Linea Especial, Nutrición y Obesidad (University of Navarra), and Carlos III Health Institute (CIBER project, CB06/03/ 1017). The authors acknowledge the scholarships provided by the Asociación de Amigos de la Universidad de Navarra to T. R.-U. and by the FPU 'Formación de Profesorado Universitario' Program of the Spanish Ministry of Education, Culture and Sport to S. G.-C.

The authors' contributions are as follows: J. A. M., M. C. A.-S., M. C. and M. O. designed and conducted the research, and reviewed the paper; T. R.-U. and S. G.-C. conducted the research, analysed the data, and wrote the paper; P. G.-M. and F. I. M. analysed the data and reviewed the paper; A. M. designed and conducted the research, reviewed the paper, and had primary responsibility for the final content. All authors read and approved the final manuscript.

None of the authors has any conflict of interest to declare.

\section{References}

1. de Onis M, Blossner M \& Borghi E (2010) Global prevalence and trends of overweight and obesity among preschool children. Am J Clin Nutr 92, 1257-1264.
2. NAOS strategy (2011) Estudio de prevalencia de obesidad infantil "ALADINO" (Alimentación, Actividad Física, Desarrollo Infantil y Obesidad) (Study on the prevalence of childhood obesity "ALADINO" (Nutrition, Physical Activity, and Obesity Child Development)). http://www.naos.aesan. msc.es/naos/ficheros/investigacion/ALADINO.pdf (accessed 20 June 2012).

3. Moleres A, Ochoa MC, Rendo-Urteaga T, et al. (2012) Dietary fatty acid distribution modifies obesity risk linked to the rs9939609 polymorphism of the fat mass and obesityassociated gene in a Spanish case-control study of children. Br J Nutr 107, 533-538.

4. Moreno LA, Gonzalez-Gross M, Kersting M, et al. (2008) Assessing, understanding and modifying nutritional status, eating habits and physical activity in European adolescents: the HELENA (Healthy Lifestyle in Europe by Nutrition in Adolescence) study. Public Health Nutr 11, 288-299.

5. Baker JL, Olsen LW \& Sorensen TI (2008) [Childhood body mass index and the risk of coronary heart disease in adulthood]. Ugeskr Laeger 170, 2434-2437.

6. Wabitsch M (2000) Overweight and obesity in European children: definition and diagnostic procedures, risk factors and consequences for later health outcome. Eur J Pediatr 159, Suppl. 1, S8-S13.

7. Reinehr T \& Wabitsch M (2011) Childhood obesity. Curr Opin Lipidol 22, 21-25.

8. Owen CG, Martin RM, Whincup PH, et al. (2005) Effect of infant feeding on the risk of obesity across the life course: a quantitative review of published evidence. Pediatrics 115, 1367-1377.

9. Crocker MK \& Yanovski JA (2011) Pediatric obesity: etiology and treatment. Pediatr Clin North Am 58, 1217-1240, xi.

10. Katz DL, O'Connell M, Yeh MC, et al. (2005) Public health strategies for preventing and controlling overweight and obesity in school and worksite settings: a report on recommendations of the Task Force on Community Preventive Services. MMWR Recomm Rep 54, 1-12.

11. Ford AL, Hunt LP, Cooper A, et al. (2010) What reduction in BMI SDS is required in obese adolescents to improve body composition and cardiometabolic health? Arch Dis Child 95, 256-261.

12. Lavelle HV, Mackay DF \& Pell JP (2012) Systematic review and meta-analysis of school-based interventions to reduce body mass index. J Public Health (Oxf) 34, 360-369.

13. Burczynski ME \& Dorner AJ (2006) Transcriptional profiling of peripheral blood cells in clinical pharmacogenomic studies. Pharmacogenomics 7, 187-202.

14. Caimari A, Oliver P, Keijer J, et al. (2010) Peripheral blood mononuclear cells as a model to study the response of energy homeostasis-related genes to acute changes in feeding conditions. OMICS 14, 129-141.

15. Sanchez J, Priego T, Pico C, et al. (2012) Blood cells as a source of transcriptional biomarkers of childhood obesity and its related metabolic alterations: results of the IDEFICS study. J Clin Endocrinol Metab 97, E648-E652.

16. Bouchard L, Rabasa-Lhoret R, Faraj M, et al. (2010) Differential epigenomic and transcriptomic responses in subcutaneous adipose tissue between low and high responders to caloric restriction. Am J Clin Nutr 91, 309-320.

17. Crujeiras AB, Parra D, Milagro FI, et al. (2008) Differential expression of oxidative stress and inflammation related genes in peripheral blood mononuclear cells in response to a low-calorie diet: a nutrigenomics study. OMICS $\mathbf{1 2}$ 251-261.

18. Radom-Aizik S, Zaldivar F Jr, Leu SY, et al. (2009) A brief bout of exercise alters gene expression and distinct gene 
pathways in peripheral blood mononuclear cells of earlyand late-pubertal females. J Appl Physiol 107, 168-175.

19. Radom-Aizik S, Zaldivar F Jr, Leu SY, et al. (2009) Brief bout of exercise alters gene expression in peripheral blood mononuclear cells of early- and late-pubertal males. Pediatr Res 65, 447-452.

20. Cole TJ, Bellizzi MC, Flegal KM, et al. (2000) Establishing a standard definition for child overweight and obesity worldwide: international survey. BMJ 320, 1240-1243.

21. Rendo-Urteaga T, Garcia-Calzon S, Martinez-Anso E, et al. (2013) Decreased cardiotrophin-1 levels are associated with a lower risk of developing the metabolic syndrome in overweight/obese children after a weight loss program. Metabolism 62, 1429-1436.

22. Rendo-Urteaga T, Puchau B, Chueca M, et al. (2014) Total antioxidant capacity and oxidative stress after a 10-week dietary intervention program in obese children. Eur J Pediatr 173, 609-616

23. Santacruz A, Marcos A, Warnberg J, et al. (2009) Interplay between weight loss and gut microbiota composition in overweight adolescents. Obesity (Silver Spring) 17, 1906-1915.

24. Schofield WN (1985) Predicting basal metabolic rate, new standards and review of previous work. Hum Nutr Clin Nutr 39, Suppl. 1, 5-41.

25. Moreno LA, Ochoa MC, Warnberg J, et al. (2008) Treatment of obesity in children and adolescents. How nutrition can work? Int J Pediatr Obes 3, Suppl. 1, 72-77.

26. Moreno LA, Mesana MI, Gonzalez-Gross M, et al. (2006) Anthropometric body fat composition reference values in Spanish adolescents. The AVENA Study. Eur J Clin Nutr 60, 191-196.

27. Marques M, Moleres A, Rendo-Urteaga T, et al. (2012) Design of the nutritional therapy for overweight and obese Spanish adolescents conducted by registered dieticians: the EVASYON study. Nutr Hosp 27, 165-176.

28. Moleres A, Rendo-Urteaga T, Zulet MA, et al. (2012) Obesity susceptibility loci on body mass index and weight loss in Spanish adolescents after a lifestyle intervention. $J$ Pediatr 161, 466.e2-470.e2.

29. Matthews DR, Hosker JP, Rudenski AS, et al. (1985) Homeostasis model assessment: insulin resistance and $\beta$-cell function from fasting plasma glucose and insulin concentrations in man. Diabetologia 28, 412-419.

30. Katz A, Nambi SS, Mather K, et al. (2000) Quantitative insulin sensitivity check index: a simple, accurate method for assessing insulin sensitivity in humans. J Clin Endocrinol Metab 85, 2402-2410.

31. Cinar MU, Islam MA, Pröll M, et al. (2013) Evaluation of suitable reference genes for gene expression studies in porcine PBMCs in response to LPS and LTA. BMC Res Notes $\mathbf{6}, 56$.

32. Wang T, Liang ZA, Sandford AJ, et al. (2012) Selection of suitable housekeeping genes for real-time quantitative PCR in
CD4(+) lymphocytes from asthmatics with or without depression. PLOS ONE 7, e48367.

33. Irizarry RA, Hobbs B, Collin F, et al. (2003) Exploration, normalization, and summaries of high density oligonucleotide array probe level data. Biostatistics 4, 249-264.

34. Dennis G Jr, Sherman BT, Hosack DA, et al. (2003) DAVID: Database for Annotation, Visualization, and Integrated Discovery. Genome Biol 4, P3.

35. Huang da W, Sherman BT \& Lempicki RA (2009) Systematic and integrative analysis of large gene lists using DAVID bioinformatics resources. Nat Protoc 4, 44-57.

36. Huang da W, Sherman BT \& Lempicki RA (2009) Bioinformatics enrichment tools: paths toward the comprehensive functional analysis of large gene lists. Nucleic Acids Res 37, $1-13$.

37. Bouwens M, Afman LA \& Muller M (2008) Activation of peroxisome proliferator-activated receptor $\alpha$ in human peripheral blood mononuclear cells reveals an individual gene expression profile response. BMC Genomics 9, 262.

38. Oana F, Takeda H, Hayakawa K, et al. (2005) Physiological difference between obese $(\mathrm{fa} / \mathrm{fa})$ Zucker rats and lean Zucker rats concerning adiponectin. Metabolism $\mathbf{5 4}$, 995-1001.

39. Lumeng CN, Deyoung SM, Bodzin JL, et al. (2007) Increased inflammatory properties of adipose tissue macrophages recruited during diet-induced obesity. Diabetes 56, 16-23.

40. El-Hagracy RS, Kamal GM, Sabry IM, et al. (2010) Tissue factor, tissue factor pathway inhibitor and factor VII activity in cardiovascular complicated type 2 diabetes mellitus. Oman Med J 25, 173-178.

41. Skopkova M, Penesova A, Sell H, et al. (2007) Protein array reveals differentially expressed proteins in subcutaneous adipose tissue in obesity. Obesity (Silver Spring) 15, $2396-2406$

42. Matsuzawa Y (2005) White adipose tissue and cardiovascular disease. Best Pract Res Clin Endocrinol Metab 19, 637-647.

43. Macias MP, Gerkin RD \& Macias JD (2010) Increased amphiregulin expression as a biomarker of cholesteatoma activity. Laryngoscope 120, 2258-2263.

44. Fukatsu Y, Noguchi T, Hosooka T, et al. (2009) Musclespecific overexpression of heparin-binding epidermal growth factor-like growth factor increases peripheral glucose disposal and insulin sensitivity. Endocrinology 150, 2683-2691.

45. Ogunwobi OO \& Beales IL (2008) Leptin stimulates the proliferation of human oesophageal adenocarcinoma cells via HB-EGF- and Tgf $\alpha$-mediated transactivation of the epidermal growth factor receptor. BrJ Biomed Sci 65, 121-127.

46. Vereecke L, Beyaert R \& van Loo G (2009) The ubiquitinediting enzyme A20 (TNFAIP3) is a central regulator of immunopathology. Trends Immunol 30, 383-391.

47. Jenner RG \& Young RA (2005) Insights into host responses against pathogens from transcriptional profiling. Nat Rev Microbiol 3, 281-294. 